Les expérierices faites entre temps sur le canal d'amenée avaient démontré que sa pente était suffisante et qu'avec un dessableur du nouveau lype l'ensablement ne se produrait plus.

Les aväntages de ce nouveau type de dessableur allaient donc ctre : de supprimer l'ensablement du canal d'amenée, d'augmen. ter de façon intense lc dessablage de l'eau et de réduire considérablement les frais de cette opération puisque la manutention des dessableurs ne comporteralt plus que la survellance générale ct to nelloyage de la grille. Pour les turbines, on pouvail s'allendre à une longévité plus grande des pièces soumises à l'usure et à une plus grande sécurité du-service, avantages très précieux dans un pays comme le Chıli où les pièces de réserve doivent ètre tirées d'Europe et où le personnel capable de réparer des turbines est difficile à obtenir.

Ces avantages fort plausıbıes en eux-mèmes étaient cependant difficiles à exprimer par des chiffres qui eussent pu justifier de façon toute objective la dépense et le risque que comportait la construction des deux grands dessableurs d'un système perfectionné, apparemmeni compliqué et complètement nouveau.

$$
\text { (4 suivie.) }
$$

Henri Dufour, Ingénieur.

\section{L'UTILISATION DES COMBUSTIBLES}

\section{Comment les usines à gaz peuvenc parer à la pénurie du charbon (1).}

A la sulte des difficultés de transport, un grand nombre d'usines à gaz ont dû arrêter leur production ces derniers temps. Par: mi celles-ci, signalons celles de Carpentras, Tourmon, Bagnols, Paray-le-Monial, Cours, etc... Comme il semble bien que cello pénurie doive ètre, pendanl quelques années encore, considérée comme un étal endémique, il semble prudent pour les usines a gaz de s'outiller pour y parer dans la mesure du possible. Nous voudrions indiquer aujourd'hui un orocédé permetlant d'augmenter considérablement le readement du charbon en gaz sans modilications bien considérables. Il consiste essentıellement à empêcher la décomposition des hydrocarbures qui habituellement sćjournent dans la cornue un temps trop considérable au contact des parois portées à haute température. Le résultat immédiat est un dépòt compact de charbon dit de cornue qui, tout en diminuant la richesse du gaz produit, forme un enduit isolant, augmente l'épaisseur de la cornue et oblige à employer, pour le chauflage, une quantité excessive de coke.

Ce balayage s'opère au moyen d'un mince filet de vapeur surchauffée introduit, s'il s'agit de cornues horizontales, par l'arrière de celles-ci. Cette vapeur, au contact du charbon, produit du gaz à l'eaì lequel, par son volume, dilue les hydrocarbures et les entraine rapidement hors de la cornue. Des essais que nous avons pratiqués, il résulte que le réndement en gaz passe de $300-350$ mètres cubes à 550-65o mètres cubes à la tónne, sans que pour cela le pouvoir calorifique du gaz soit abaissé dans des proportions excessives. Il conserve au mètre cube de 4.200 à 4.400 calories au lieu de 4.800 que donne la distillation, habituellement pratiquée avec les charbons dont on peut disposer actuéllement. On parvient, ainsi à extraire d'une tonne de houille environ 2.500 .000 calories sous forme de gaz au lieu de 1.250 .000 calories qui constituent un -rendement normal.

On s'explique 'la richesse du' gaz ainsi obtenu par le ròle protecteur que joue le gaz à l'eau vis-à-vis des carbures benzéniques et méthaniques. Ceux-ci, habituellement se décomposent sous l'influence d'une haute température,- en-donnant naissance au charbon de cornue, au gràphite, à la naphtaline, à l'anthracène et autres carbures à poids moléculaires élevés dont on connaît l'influence désastreuse dans l'entretien des tuyauteries où ils se déposent. Ce sont les carbures légers qui enrichissent suffisamment le gaz pour lui permettre un pouvoir calorique encore élevé On observe d'autre part la formation de goudrons beaucoup plus fluides et la diminution de la proportion de brai. On peut comp-

(1) Extrait de la Revue industrielle de l'Est. ter sur ume moyeune de 5 i $6 \%$ de goudron. Cette quantité, dans un four à sept cornues bren dirigé. permet son chauffage à peu près complet par l'emplor d un bon brùleur à goudron, fonctionnanl avec un très faible excès d'uir. On an, constale jamais aucun encrassement des cornues, mass il ęst certan que l'on perd, par suite de la porosité de celles-cl, une petıte quantıte d'hydrogène.

br, a la sulte du lour, on a som de piaces unc recuperation d'air chaud ansi quine petite chaudice, 11 est pussible de produne toute la vapeur necessane au lonchonnement de l'usme a gat el ae ne labser sortir les lumees qu a jo0" au lieu de 1,000 is

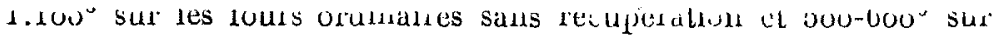

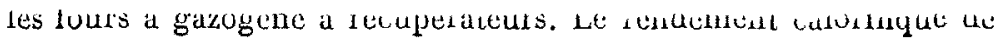

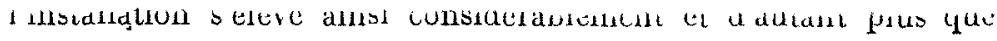
lon prend, dans ld constiuction du luar, ues precaluons a 150 lement caloriaque ventabenent trop hughoes habltatiement.

un autce arallage au procede est a upe. el automaliquemenc lo netroyage du coke de sa poussiere. 1 res liequimment, arej les noulles deplorables que 1 on dut distiller peildant la guere, il arrivait que te coke onlenu étall très poussiereux. Il l'elail d'autaut plus que 1 on completant les chargen de connues avec de la scrure et des debris de boss. Ur, l'emploi d une injecion contirue de vapeur permet la destruction de tout ce poussicr qui se trouve le premier altaqué. un peut done, a la rigueur, oblenrr un mème rendement en coke quautrelors avec ce procédé si l'on a soin de mélanger la houlle d'une quantité de sclure sufinsante pour que le chiarvon de boss formé puisse décomposer la quantir de vaperr d'eau introdute.

A notre connassance, ce procédé trop peu connu en Filince, 1: a été employé sur cornues horizontales que daus une seule usine. 11 est en montage dans une seconde usine. Par contre, en Angleterre, on laurait depuss peu appliqué à de nombreuses connues verticales et les résullats qu'il donne amsı sont absolument identiques à ceux que nous avons obtenus nous-memes.

Ajoutons que l'application de ce procedé demande beaucoup de soins, pour éviter de trop refrondır les fonds de cornue ce qui pourrait entraîner leur crevassemcnt. Bien applıquë, il permet des économies de charbon très élevées et fournil la même quantité de coke que la distillation sans vapeur, étant donné l'énorme augmentation de production de chaque four el la réduction concomittante du coke employé pour le chauliage. Enfin, par l'emploi du brûleur à goudron, on simplitie singulièrement la surveillance du chaulfage des fours toujours délncate de nuit et l'on peut réduire la mam-d'œuvre à sa plus simple expression.

Dans le cas du chaufage au coke, on peut améliorer considérablement le rendement par l'emploi de foyers divergents formant une chambre de combustion plus vaste avec voûte de grand sayon. On peut également, très simplement, obtenir le réchauffage de l'air einployé a la combustion. On fait pénétrer celui-ci par l'arrière du cendrier, ce qui permet do lui faire parcourir uñe galerie étroite à chucanes où il s'échauffe all contact des fumées quittant le four par le carneau se rendant à la chcminée. Les foyers devieunent blancs et les grilles très épaisses se ramollissent et se déforment très vite si l'on n'a soin d'alimenter le cendrier en eau Celui-ci cst, bien entendu, hermétiquoment clos a l'avant.

Il semble que dans la généralité des cas, avoc une diminution concomiltante du prix du mire cube, les villes accepteraient ce mode de fabrication Etant donné même la pénuric du charbon en France, nous estimons que l'emploi de ce procédé s'impose, parce que plus économique que tont antre, il peut ètre installé sans grandes modifications sur tous les genres de fours.

Ajoutons qu'il permet l'emploi de houjlles très médiocres at même de charbons barrés. Toulefois le pouvoir calorifique du gaz obtenu s'abaisse sensiblement avec la qualité du combustible.

H. Contaut.

\section{La récupération de l'énergie sur les chemins de fér} electriques à la descente des pentes $\left(^{1}\right)$.

Dans les circonstances actuelles, où les économies en combus. tible s'imposent d'une façon sí intense, la question de la récupé-

(1) Extrait de la Revue indusirielle de l'Esl. 
ration de lénergie sur les chemins de fer électriques à la descente des pentes prend unc importance particulière.

Cette récupération a déjà été obtenue sur certains réseaux de tramways électriques. Différents systèmes ont été appliqués.

Le mojen le plus simple consiste à employer le moleur asynchronc. Un sait, en elfet, qu'un moteur à champ tournant à rotor bobiné ou à cage d'ícurcuil devient automatiquement générateur lorsqu'on le fait tourner à une vitesse supérıeure à celle du synchronisme. Le procédé a été appliqué avec succès en Italie. Mais il nécessite un aménagement spécial de la station gézératrice et de la ligne, pour que l'afflux du courant récupéré ne trouble pas la distribution.

L'emploi du courant continu semble rendre facile, à priori, cette récupération, mais, dans la pratique, les résultats in ont pas donné ce qu'on attendait. Toutelois, des essais assez satisfaisants ont été obtenus sur le Métropolitain, à Paris.

La traction par courant monophasé se prête moins facilement à la récupération de l'énergic potenticlle aux descentes, on y parvient cependant facilement en installant sur la locomotive un convertisseur transformant le courant monophasé en courant triphasé. Mais c'est là une complication qui ne doit être admise que dans des cas particuliers

\section{REVUE DES SOCIETES SAVANTES}

\section{ET DES PUBLICATIONS SCIENTIFIQUES}

\section{SOCIÉTÉ HYDROTECHNIQUE DE FRANGE}

Le Comité de la Société hydrotechnique de France a tenu récemment une réunıon dans laquelle diverses question fort intéressantes au pöint de vue de l'hydraulique, ont été soulevées. Nous les résumons ici.

$I^{\circ}$ La demande de subvention à l'Etat permettant l'aménagement du laboratoire dessais des turbines de Beauvert, a reçu un accueil favorable de la paut du Comité d'Etudes et de Recherches scientifiques pour l'utilisation et l'aménagement des forces hydrauliques. Rappelons que ce laboratoire devra toujours rester ouvert aux inventeurs ou constructeurs désireux de se livrer à des recherches personnelles.

Uné commission spéciale, chargée d'étudier la nature et l'ordre des essais à effectuer dans ce laboratoire aux frais de la Société hydrotechnique elle-même, est ainsi composée : MM. Rateau, Eydoux, Barbillion, A. Bouchayer, Gariel, Boucher, Bouvier, de la Brosse, Camichel, Halin, Jouguet, Mariès, Pérot, de Sparre.

$2^{\circ}$ Les désirs des constructeurs, en ce qui conceme les pertes de charges, ont été résumés en un programme soumis au Comité. En ce qui concerne les essais de turbine, lo programme est également fait, mais le laboratoire n'est pas achevé.

$3^{\circ}$ La commission des réseaux a établi les cartes aù $200.000^{\circ}$ concernant les installations électriques existani en France. Le détail en est donné ci-dessous.

Les cartes au 500 ooo sont également en venle

$4^{\circ} \mathrm{M}$. Clemens Herschel, auteur du jaugeur Venturi, a communiqué à la Société des rechcrches faites sur un nouveau type de déversoir ; les règles d'ćtablissement de ce déversoir sont les suivantes: Amener l'eau sur un radier incliné de ${ }_{2} / \mathrm{T}$ a une crête formée par une surfacc cylindrique creuse ; l'cau après avoir franchi cette surface, s'écoulerait sur un autre radier également incliné de $2 / \mathrm{r}$, mais en sens contraire. On mesurerait la charge sur la crête et quelque part en amont, un verrait ensuite si le débit ne se rattacherait pas par une loi quelconque, soit à la charge sur la crête, soit à la différence des charges sur cette crête et à l'amiont. En fait, le volume écoulé jar unité de largeur du "déversoir se tronve être une fonction linćaje de la différence do ces charges.

La prise de pression a la crête se faisait au moyen de trous de
$3 \mathrm{~mm}$. r/2 de diamètre, dont l'axe était normal à la paröi d'amenée de l'eau au point où cellc-ci se raccorde tangentiellement à la crête.

Le facteur numérique qui alfecte la différence des pressions est donné comme égal à 5,5 , toutes mesures cxprimées en valeur anglaise : avec dos mosures françaisos, il serait par mètre courant, égal à $\mathrm{x}, 675$. Il eût été désirable que les expóriences eussent été poursuivies et, comme le dit l'anleur lui-mème, qu il eût été possıble de délerminer quelle est, sur le facteur numérique de sa formule, l'influence du rayon de la crète, ce rayon étant de fo millmètres, mais on avait atteint la date du 3 octobre, et l'Université du Massachusets, où s'opéraient ces recherches, rouvrait ses portes aux élèves le lundi 6 , le savant hydraulicien dut déménager et enlever son outillage on toute hâtc.

$5^{\circ}$ Enfin, M. de Sparre annonça l'envoi d'ume étude sur les turbines centripètes à réaction et les condilions à remplir pour réduire au minimum la diminution du rendement dans le cas, soit d'une diminution de la charge, soit d'une variation de la hauteur de chute.

La solution du problème dépend de certains coefficients dont l'auteur a pris la raleur un peu arbitrairement en atteridant qu'ils fussent établis par l'expérience.

Cartes aU 200.000

\begin{tabular}{|c|c|c|c|c|c|c|c|c|}
\hline \multirow{2}{*}{$\begin{array}{c}\text { No } \\
\text { des } \\
\text { cartes }\end{array}$} & \multicolumn{2}{|c|}{ Prix des cartes } & \multirow{2}{*}{$\begin{array}{c}\text { No } \\
\text { des } \\
\text { cartes }\end{array}$} & \multicolumn{2}{|c|}{ Prix des cartes } & \multirow{2}{*}{$\begin{array}{c}\text { No } \\
\text { des } \\
\text { cartes }\end{array}$} & \multicolumn{2}{|c|}{ Prix des cartes } \\
\hline & en noir & $\begin{array}{c}\text { en } \\
\text { couleur }\end{array}$ & & ell noir & $\begin{array}{c}\text { en } \\
\text { conltur }\end{array}$ & & en noir & $\begin{array}{c}\text { en } \\
\text { conleur }\end{array}$ \\
\hline 1 & 3,10 & $\Rightarrow$ & 28 & 'trasbourg & & 55 & 5,10 & $\eta$ \\
\hline 2 & 5,10 & 6,00 & 29 & 5,10 & $\eta$ & $: 6$ & 5,10 & 11,10 \\
\hline 3 & 2,40 & 3,90 & 30 & 5,10 & 6,60 & 57 & 5,10 & 9,00 \\
\hline 4 & \multicolumn{2}{|c|}{ En prépardtion } & 31 & 5,10 & 9,00 & 58 & 5,10 & 6,0 \\
\hline 5 & \multicolumn{2}{|c|}{ En préparatıon } & 32 & $5,10^{-}$ & 9,00 & 59 & 5,10 & 9,60 \\
\hline 6 & 5,10 & 5,46 & 33 & 5,10 & 8,10 & 60 & 5,10 & 11,10 \\
\hline 7 & 5,10 & 6,09 & 34 & 5,10 & 7,50 & 61 & 5,10 & $5,4,0$ \\
\hline 8 & 5,10 & 6.60 & $35^{\circ}$ & 5.10 & 20,10 & 62 & 2.40 & 70 \\
\hline 9 & \multicolumn{2}{|c|}{ En préparation } & $3 i$ & Mulhouse & $\begin{array}{l}\text { Pas de } \\
\text { renseig }\end{array}$ & 63 & 5,10 & 6,00 \\
\hline 10 & 5,10 & 6,90 & 37 & 5,10 & $\begin{array}{r}\text { rensig } \\
7,50\end{array}$ & 64 & 5,10 & 7,50 \\
\hline 11 & 5,10 & 5,40 & 38 & 5,10 & 6,60 & 65 & 5,10 & 7,50 \\
\hline 12 & 5,10 & v & 39 & 5,10 & 6,60 & 66 & 5,10 & 12,60 \\
\hline 13 & 5,10 & $\eta$ & 40 & 5,10 & 5,60 & 67 & 5,10 & 9,00 \\
\hline 14 & 5,10 & $\dot{n}, 00$ & 41 & 5,10 & 17,50 & 8 & 5,10 & 9,10 \\
\hline 15 & 5,10 & 11,10 & 42 & 5.10 & 17,10 & 69 & 2,40 & 6,00 \\
\hline 16 & 5,10 & 18,60 & 43 & 2,40 & 3,70 & 70 & 5,10 & 10.50 \\
\hline 17 & 5,10 & 750 & $4 \hat{q}$ & 5,10 & 6,00 & 71 & 5,10 & 11,10 \\
\hline 18 & Melz & Pas de & 45 & 5,10 & 8,10 & 72 & 5,10 & 17,10 \\
\hline 19 & Saverne & renseig. & 46 & 5,10 & 9,60 & $73^{\circ}$ & 5,10 & 11,10 \\
\hline 20 & & 7,50 & 47 & 5,10 & 12,50 & 74 & 5,10 & 15,00 \\
\hline 21 & & 6,00 & 48 & 5,10 & 17,10 & 75 & 2,40 & 3,90 \\
\hline 22 & 5,10 & 6,00 & 49 & 5,10 & $»$ & 76 & 5,10 & 5,40 \\
\hline 23 & 5,10 & 6,00 & 50 & 5,10 & 7,50 & 77 & 5,10 & 6,60 \\
\hline 24 & 5,10 & 6,00 & 51 & 5,10 & 9,00 & 78 & 5,10 & 14,10 \\
\hline 25 & & 8,10 & 52 & 5,10 & $\dot{9}, 60$ & 79 & \multicolumn{2}{|c|}{ Corse } \\
\hline 26 & 5,10 & 6,60 & $5 ?$ & 5,10 & 20,10 & 80 & \multicolumn{2}{|c|}{ Corse } \\
\hline 27. & \multicolumn{2}{|c|}{ Enpréparation } & 54 & 5,10 & 20,10 & 81 & \multicolumn{2}{|c|}{ Cor'sé } \\
\hline
\end{tabular}

Prix de la collection complète en noir..... $340 \mathrm{fr}$. 80 " $)$ ij. en couleur". $584 \mathrm{fr} .7^{\circ}$

Cartes aU $500.000^{*}$ PRIX DE VENTE

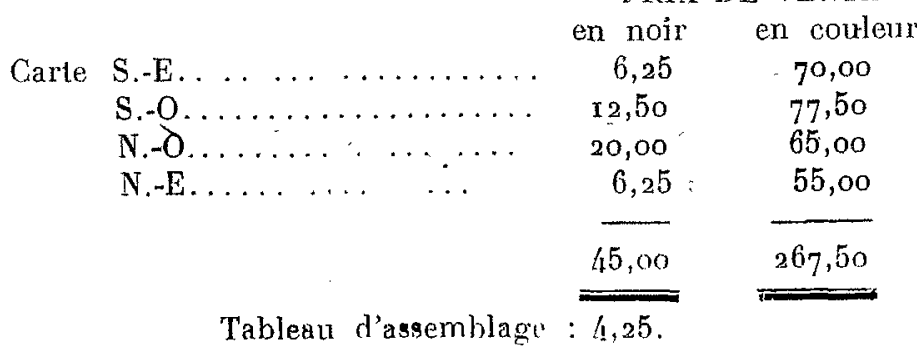

